\title{
Skin Health Properties of Lycopene and Melatonin
}

\section{Lourdes Franco ${ }^{*}$, Ana M Marchena², Ana B Rodríguez ${ }^{2}$}

'Department of Physiology (Neuroimmunophysiology and Chrononutrition Research Group), Faculty of Medicine, University of Extremadura, Badajoz, Spain ${ }^{2}$ Department of Physiology (Neuroimmunophysiology and Chrononutrition Research Group), Faculty of Science, University of Extremadura, Badajoz, Spain.

\section{Article Info}

\section{Article Notes}

Received: December 28, 2020

Accepted: March 17, 2021

\section{${ }^{*}$ Correspondence:}

*Dr. Lourdes Franco, Department of Physiology, Faculty of Medicine, University of Extremadura, Avda. Elvas s/n, 06006 Badajoz, Spain; Email: lourdesfh@unex.es.

${ }^{0} 2021 \mathrm{Franco} \mathrm{L}$. This article is distributed under the terms of the Creative Commons Attribution 4.0 International License.

\section{Keywords:}

Antioxidant

Lycopene

Melatonin

Skin aging

\section{Abstract}

Skin plays an important role in the protection of our body. It can be damaged by environmental factors, and it suffers from progressive morphological and physiological disorders with time. Melatonin and Lycopene have a lot of properties which protect our skin. In this review, we have investigated about how these substances can help to prevent damage and repair the skin.

\section{Review}

Skin plays an important role in the thermoregulation of the body, as it regulates the interchange of water and electrolytes with the environment ${ }^{1}$. It also acts as a barrier: a physical filter that absorbs ultraviolet (UV) radiation, a mechanical anti-trauma obstacle and a biological hurdle against microorganisms ${ }^{2} \mathrm{UV}$ radiation is a strong component that is capable of generating ROS at the skin level. There are several types of ROS and their generation depends on the UV wavelength. Type B UV radiation produces the production of 02 a by activating NADPH oxidase and respiratory chain reactions, while type A UV radiation produces 02 a because it performs a photosensitizing reaction with internal chromophores such as riboflareactio porphyrin ${ }^{3}$. In a specific cell type, ROS stimulates cell growth. A high level of superoxide anion is required for phorbol ester-induced tumor promotion. Various external stress-inducing stimuli (eg, UV radiation, vanadate, and silica) lead to the release of ROS in cells, resulting in the activation of AP-1 via the MAP kinase pathway ${ }^{4}$.

Furthermore, photoaging, i.e. aging induced by UV radiation exposure, depends primarily on the degree of sun exposure and skin pigment. People who do not have an outdoor lifestyle, but live in sunny climates have less melanin. In consequence, they will experience a greater degree of photoaging ${ }^{5}$.

However, skin can be damaged; it suffers from progressive morphologic and physiologic disorders with time. The factors causing skin damage can be environmental due to the fact that it is constantly exposed to air, solar radiation and other environmental agents. Mechanical and chemical damage can induce the generation of free radicals and reactive oxygen species (ROS), leading to an imbalance in favour of pro-oxidant systems causing oxidative stress with pathological implications ${ }^{5}$.

The above-mentioned free radicals are chemical species possessing an unpaired electron that can be considered as fragments of molecules which are generally highly reactive. They are continuously produced in cells either as accidental by-products 
of metabolism or deliberately during phagocytosis, for example. Oxygen and its radical derivatives are some of the most harmful chemical reagents in terms of the damage they can produce in aerobic cells ${ }^{1}$.

Thus, in a person's daily life, their skin is constantly being damaged by reactive species derived from endogenous processes, as well as environmental agents. This damage is repaired by cellular mechanisms, but if the frequency of injurious events surpasses the rate of repair, damage may turn into permanent and irreversible $e^{1,2}$. One of the most damaging environmental factors for the integrity of the skin, as hinted above, is UV radiation, which promotes skin aging, as it leads to formation of free radicals, which ultimately causes a loss of skin elasticity and reduces the ability of the skin to retain water ${ }^{6}$. Therefore, avoiding sun exposure and making use of sunscreen reduces the risk of cancer and skin aging.

Not only does skin reveal the signs of aging caused over time, but also the physiological aging due to environmental factors. Dryness, irregular pigmentation, redness appearance, loss of elasticity and deep wrinkles are some of the visible signs on aged skin. During the last years, substantial progress has been made in understanding cellular and molecular mechanisms that bring about chronological aging and photoaging. The latest research reveals that chronological aging and photoaging share fundamental molecular pathways ${ }^{5,7}$.

As it is known, the exogenous antioxidants are substances contained in food that favor the oxidationreduction reactions balance in cell metabolism. Among these, there are three phytochemicals widely mentioned in the literature: carotenoid lycopen, resveratrol stilbene and vitamin $\mathrm{C}$. The chemical structure of carotenoids determines their physical properties, chemical reactivity and biological activity; on the other hand, their nine or more conjugated bonds make them function as efficient ROS scavengers ${ }^{8}$. Lycopen is typically found in tomato, as well as other red fruits and nutritional supplements ${ }^{9}$.

The skin dermal extra-cellular matrix consists of collagen type I and III, hyaluronan and chondroitin dermatan sulfate as major components. Among them, chondroitin dermatan sulfate appeared to be a possible marker for the tissue fibrosis, which is a characteristic of the photodamage acid has both radical scavenging activity and iron chelating activity. Reactive oxygen species are thought to be associated with the wrinkling due to photodamages of the skin induced by exposure to UV irradiation. Especially the hydroxyl radical is very damaging to a variety of biological substances ${ }^{10}$.

Since the effectiveness of endogenous antioxidant systems decreases during aging, the exogenous supplementation of antioxidants, both from dietary intake and topical application, might be a protective strategy against age-associated skin oxidative damage ${ }^{4}$. This, together with the fact that life expectancy has increased to unexpected limits and, therefore, more and more people are concerned about their appearance and looking younger, has brought a revolution in the current cosmetic industry.

The latest cosmetic generation combines natural or synthetic antioxidants with innovative formulae ${ }^{11}$. Most promising topical treatments for skin aging prevention include herbal extracts, vitamins and antioxidants food supplement, which have been widely accepted to scavenge free radicals from skin cells and to restore skin conditions. The studies about development of cosmetic products against skin aging involve making use of antioxidant substances capable to prevent and treat skin damage ${ }^{12,13}$.

Melatonin is produced mainly by pinealocytes in the pineal gland, which is located in the medline of the brain, just above the posterior commissure at the dorsal ventricle. Is also produced by neuroendocrine cells in the retinan harderian glands, gastrointestinal tract and pancreas edge of the third ventricle ${ }^{14}$ and the cosmetic industry tests a large number of this substance with sunscreen properties ${ }^{15}$. Among these substances, we find melatonin and lycopene ${ }^{16}$, which showed a clearly efficacy in the improvement of skin elasticity and pronounced hydration effects on human skin. Lycopene is an effective antioxidant, and this is clearly a major important mechanism of lycopene action. In that sense, lycopene can trap singlet oxygen and reduce mutagenesis in the Ames test. Evidence is accumulating for other mechanisms as well. Lycopene in physiological concentrations is capable of inhibiting the growth of human cancer cells because it interferes with the signals of the growth factor receptor and the cell cycle in prostate cancer cells without evidence of toxic effects or apoptosis of the cells ${ }^{17}$. The hormone melatonin is a highly conserved molecule, it has a crucial role in the maintenance of the skin. As the skin has functional melatonin receptors and also acts as a complete system capable of producing and regulating melatonin synthesis, melatonin is a promising candidate for its maintenance and protection ${ }^{18}$. Physiologically, the best known role of melatonin is that of a chronobiotic factor or zeitgeber, which is capable of regulating the oscillations of the internal biological clock. It is thought to act in the control of seasonal and circadian rhythms. This is based on the fact that melatonin secretion is related to ambient light and normally exhibits a strictly regulated diurnal pattern. In this sense, melatonin is sometimes called "the hormone of darkness"14.

Firstly, melatonin is a hormone synthesized mainly by the pineal glandule. It is also produced in other parts of the body, like by chromaffin cells in the intestines. The main function of melatonin in the body involves the control of circadian and seasonal rhythms ${ }^{19}$, but it is also 
implicated in skin functions, such as hair growth cycling or fur pigmentation and has an important role in melanoma control $^{20-23}$. Many studies report anti-oxidative properties of melatonin as a free radical scavenger ${ }^{24,25}$, due to the fact that it suppresses ultraviolet (UV)-induced damage to skin cells and shows strong antioxidant activity in UV exposed cells ${ }^{26}$. Melatonin could neutralize environmental or internal stresses to preserve the integrity of the organism and to maintain its homeostasis ${ }^{27}$. Melatonin increased collagen accumulation in the cultures molecular mechanism of melatonin promotion of self-renewal of NSCs in which a chain reaction in the ERK and TGF- $\beta$ / Smad pathways promotes self-renewal and transcription of nestin ${ }^{28}$. Tamura $\mathrm{H}$ et $\mathrm{al}^{29}$, reported that melatonin has an important function in the complex endogenous control of HF biology, not only as an antioxidant function, but also inhibit keratinocyte apoptosis in the short-term on mice skin $^{30}$.

Secondly, lycopene is a pigment from carotenoids family that lacks provitamin A activity; however, it has potent antioxidant properties ${ }^{31}$. It is responsible for the red colour in several fruits and vegetables, such as tomato or watermelon, and it is also synthesized by some microorganisms. Some studies have shown that lycopene has anticancer properties against certain tumours like colon, prostate, lung or breast ${ }^{32-34}$. Moreover, its chemopreventive effects against photo-induced tumours in mice models have also been reported. In other studies, topical application of lycopene before UV radiation exposure reduced photodamage in a dose-dependent relationship ${ }^{35,36}$. Most of the reported health benefits of lycopene are attributed to its ability to protect cells against oxidative damage. Despite the fact that the amount of research focused on lycopene has not been as large as that focused on other carotenoids, on in-vitro studies lycopene appears to be a very efficient quencher of singlet oxygen and a potent scavenger of oxygen radicals ${ }^{37,38}$. The chemopreventive effect of lycopene against photoinduced cell damage has been proved in several studies. For this reason, this carotenoid is already included in diets and numerous dermatological products ${ }^{39-41}$.

Some studies reported the benefits of consuming virgin lycopene-enriched olive oil for the antioxidant capacity ${ }^{11,41}$, highlighting how this compound could be a valuable tool to have in mind. Therefore, including lycopene in our daily intake could be a good way for health promotion against multiple disorders in which oxidative stress plays an important function. Adding up, lycopene is being widely employed in cosmetic formulations due to its protective properties against photodamage and skin aging ${ }^{24}$.

As for melatonin, also known chemically as N-acetyl5-methoxytryptamine, it is a natural compound found in animals, plants and microorganisms. In humans, melatonin signal forms part of the system that regulates the circadian rhythm and the sleep-wake cycle by chemically causing drowsiness and lowering the body temperature ${ }^{25,26}$. Besides its function as a synchronizer of the biological clock, melatonin also exerts a powerful antioxidant activity. Melatonin is implicated in skin functions such as hair growth cycling, fur pigmentation, melanoma control. Melatonin receptors are expressed in several skin cells including normal and malignant. It is also able to suppress ultraviolet induced damage to skin cells and shows strong antioxidant activity in UV exposed cells. The evidence suggests that melatonin could be a significant contributor to regulation of the local system that preserves the physical and functional integrity of the skin ${ }^{19}$.

In another study, Franco said the resveratrol treatment can prevented the increase in oxo8dG induced by $\mathrm{KBrO3}$, with the resveratrol treatment and the control group. The antioxidants melatonin and vitamin $\mathrm{E}$ and the spintrapping compound PBN also decreased the level ofoxo8dG in the kidney with respect to the $\mathrm{KBrO3}$ group, although the protection was partial in this case since oxo8dG was significantly lower than in the $\mathrm{KBrO} 3$ group, but it was still significantly higher than in the control group ${ }^{42}$.

In the study conducted by Marchena, a cream was formulated containing both antioxidants under study in a separate manner. The results show us that the cream significantly increases the stratum corneum elasticity and enhances the hydration conditions of the skin after the 8-week period application, when compared with the control values. The formulation containing lycopene increased pigmentation index values, so it seems to be an excellent tool to avoid skin photodamage due to its photoprotective effect on $\operatorname{skin}^{16}$.

According to the results obtained by Marchena, it can be concluded that melatonin and lycopene could be used as effective antioxidant agents, and they should be considered in the formulation of skincare products to avoid skin aging and promote skin appearance ${ }^{16,43}$.

In this sense, antioxidant cosmetics including melatonin and lycopene could be employed as useful products in formulations for hydration, protective and antiaging purposes $^{44,45}$.

\section{Conflict of interest Disclosures}

None reported.

\section{References}

1. Cheeseman $\mathrm{KH}$, Slater TF. An introduction to free radical biochemistry. Br Med Bull. 1993; 49(3): 481-493.

2. Zoubolis CC, Makrantonaki E. Clinical aspects and molecular diagnostics of skin aging. Clin Dermatol. 2011; 29(1): 3-14.

3. Masaki H. Role of antioxidants in the skin: anti-agin effects.J Dermatol Sci. 2010; 58(2): 85-90. 
4. Makrantonaki E, Zouboulis CC. Skin alterations and diseases in advanced age. Drug Discov Today Dis Mech. 2008; 5(2): e153-62.

5. Fisher GJ, Kang S, Varani J, et al. Mechanisms of photoaging and chronological skin aging. Arch of Dermatol. 2002; 138(11): 1462-70.

6. Hwang S, Lee JH, Park C, et al. A highly efficient organic sensitizer for dye-sensitized solar cells. Chem Commun. 2007; 21(46): 4887-9

7. Makrantonaki E, Zouboulis CC. The skin as a mirror of the aging process in the human organism-state of the art and results of the aging research in the German National Genome Research Network 2 (NGFN-2). Exp Gerontol. 2007; 42(9): 879-86.

8. Dueñas-García IE, Heres-Pulido ME, Arrellano-Llamas MR, et al Lycopene, resveratrol, vitamin and FeSO4 increase damage produced by pro-oxidant carcinogen 4-nitroquinoline-1-oxide in Drosophila melanogaster: Xenobiotic metabolism implications. Food and Chemical Toxicology. 2017; 103: 233-245.

9. Fernández-Ruiz V, Cámara M, Quintela JC. Ingredientes bioactivos de tomate: el licopeno. Nut. CLin. Diet. Hosp. 2007; 166: 36-40

10. Mitani H, Koshiishi I, Sumita T, et al. Prevention of the photodamage in the hairless mouse dorsal skin by kojic acid as an iron chelator Europena Journal of Pharmacology. 2001; 411(1-2): 169-174.

11. Garrido M, González-Flores D, Marchena AM, et al. A lycopeneenriched virgin olive oil enhances antioxidant status in humans. J Sci Food. 2013; 93(8): 1820-6

12. Lupo MP. Antioxidants and vitamins in cosmetics. Clin in Dermatol. 2001; 19(4): 467-473.

13. Korkina L. Phenylpropanoids as naturally occurring antioxidants from plant defense to human health. Cell Mol Biol. (Noisy-le-Grand). 2007; 53(1): 15-25.

14. Espino J, Pariente JA, Rodríguez AB. Role of melatonin on diabetesrelated metabolic disorders. World Journal of Diabetes. 2011;15 2(11): 82-91.

15. Grether-Beck S, Marini A, Jaenicke T, et al. Photoprotection of human skin beyond ultraviolet radiation. Photodermatol Photoimmunol Photomed. 2014; 30(2-3): 167-74.

16. Marchena AM, Franco L, Romero AM, et al. Skin Pharmacol Physiol 2020;33:237-243

17. Heber D, Lu Q. Overview of Mechanisms of Action of Lycopene. Experimental Biology and Medicine. 2002; 227(10): 920-3.

18. Rusanova I, Martinez-Ruiz L, Florido J, et al. Protective Effects of Melatonin on the Skin: Future Perspectives. 2019; 8; 20(19): 4948.

19. Reiter RJ. The melatonin rhythm: both a clock and a calendar. Experientia. 1993; 49: 654-664.

20. Reiter RJ. Pineal melatonin: cell biology of its synthesis and of its physiological interactions. Endocr Rev. 1991; 12(2): 151-80.

21. Slominski A, Tobin DJ, Shibahara S, et al. Melanin pigmentation in mammalian skin and its hormonal regulation. Physiol Rev. 2004; 84(4)1155-1228.

22. Slominski A, Fischer TW, Zmijewski MA, et al. On the role of melatonin in skin physiology and pathology. Endocrine. 2005; 27: 137-148.

23. Fischer TW, Slominski A, Tobin DJ, et al. 2008. Melatonin and the hair follicle. J Pineal Res. 2008;44(1):1-15.

24. Tan DX, Reiter RJ, Manchester LC, et al. Chemical and physical properties and potential mechanisms: melatonin as a broad spectrum antioxidant and free radical scavenger. Curr Top Med Chem. 2002; 2(2): 181-197.

25. Galano A, Tan DX, Reiter RJ. Melatonin as a natural ally against oxidative stress: a physicochemical examination. J Pineal Res. 2011; 51(1): 1-16.
26. Fischer TW, Slominski A, Zmijewski MA, et al. Melatonin as a major skin protectant: from free radical scavenging to DNA damage repair Exp Dermatol. 2008; 17(9): 713-30.

27. Poljšak B, Dahmane R. Free radicals and extrinsic skin aging. Dermatol Res Pract. 2012; 135206.

28. Gao Y, Ma L, Bai C, et al. Melatonin promotes self-renewal and nestin expression in neural stem cell from the retina. Histol Histopathol. 2019; 34(6): 645-654.

29. Tamura H, Takasaki A, Taketani T, et al. The role of melatonin as antioxidant in the follicle. Journal of ovarian Research. 2012; 5,5.

30. Kobayashi H, Kromminga A, Dunlop TW, et al. A role of melatonin in neuroectodermal-mesodermal interactions: the hair follicle synthesizes melatonin and expresses functional melatonin receptors. Federation of American Societes for Experimental Biology. 2005; 19: 12.

31. Stahl W, Sies H. Bioactivity and protective effects of natural carotenoids. Biochim Biophys Acta Mol Basis Dis. 2005; 1740(2): 101-107.

32. Levy J, Bosin E, Feldman B, et al. Lycopene is a more potent inhibitor of human cancer cell proliferation than either alpha-carotene or betacarotene. Nutr Cancer 1995; 24(3): 257-66.

33. Clinton SK, Emenhiser C, Schwartz SJ, et al. Cis-trans lycopene isomers, carotenoids, and retinol in the human prostate. Cancer Epidemiol Biomarkers Prev. 1996; 5(10): 823-33.

34. Narisawa T, Fukaura $\mathrm{Y}$, Hasebe $\mathrm{M}$, et al. Prevention of $\mathrm{N}$-methylnitrosoureae induced colon carcinogenesis in F344 rats by lycopene and tomato juice rich in lycopene. Jpn J Cancer Res. 2005.

35. Ahmad N, Gilliam AC, Katiyar Sk. Definitive role of ornithine decarboxylase in photocarcinogenesis. Am J Pathol. 2001; 159(3): 885-92.

36. Fazekas Z, Gao D, Saladi RN, et al. Protective effects of lycopene against ultraviolet Be-induced photodamage. Nutr Cancer. 2003; 47(2): 181-

37. Cantrell DJ, McGarvey TG, Trucott Tg, et al. Singlet oxygen quenching by dietary carotenoids in a model membrane environment. Arch Biochem Biophys. 2003; 412(1): 47-54.

38. Stahl W, Sies H. Antioxidant activity of carotenoids. Mol Aspects Med. 2003;24(6):345-351.

39. Stahl W, Heinrich U, Wiseman S, et al. Dietary tomato paste protects against ultraviolet light-induced erythema in humans. J Nutr. 2001; 131(5): 1449-51.

40. Stahl W, Sies H. Carotenoids and flavonoids contribute to nutritional protection against skin damage from sunlight. Mol Biotechnol. 2007; 37(1): 26-30.

41. Bogdan-Allemann I, Baumann L. Antioxidants used in skin care formulations. Skin Therapy Lett. 2008; 13(7): 5-9

42. Franco MN, Galeano-Díaz T, López O, et al. Phenolic compounds and antioxidant capacity of virgin olive oil. Food Chemistry. 2014; 163: 15: 289-298.

43. Cadenas S, Barja G. Resveratrol, melatonin, vitamin E, and PBN protect against renal oxidative DNA damage induced by the kidney carcinogen KBrO3. Free Radic Biol Med. 1999; 26(11-12): 1531-7.

44. Kelkel M, Schumacher M, Dicato M, et al. Antioxidant and antiproliferative properties of lycopene. Free radical research. 2011;45(8)925-940.

45. Moselhy S, Al mslmani M. Chemopreventive effect of lycopene alone or with melatonin against the genesis of oxidative stress and mammary tumors induced by 7,12 dimethyl(a)benzanthracene in sprague dawely female rats. Mol Cell Biochem. 2008; 319: 175-180. 